Sociologie et sociétés

SOCIOLOGIE

ET SOCIÉTÉS

\title{
Sociologie, mouvements sociaux, conduite de rupture
}

Le cas québécois

Sociology, Social movement, Discontinuity

The Case of Quebec

\section{Gabriel GAGNON}

Volume 10, numéro 2, octobre 1978

Changement social et rapports de classes

URI : https://id.erudit.org/iderudit/001272ar

DOI : https://doi.org/10.7202/001272ar

Aller au sommaire du numéro

\section{Éditeur(s)}

Les Presses de l'Université de Montréal

\section{ISSN}

0038-030X (imprimé)

1492-1375 (numérique)

Découvrir la revue

Citer cet article

GAGNON, G. (1978). Sociologie, mouvements sociaux, conduite de rupture : le cas québécois. Sociologie et sociétés, 10(2), 103-122.

https://doi.org/10.7202/001272ar

\section{Résumé de l'article}

Postulant avec Touraine une liaison nécessaire entre sociologie et mouvements sociaux, l'auteur s'interroge sur les méthodes par lesquelles ces mouvements peuvent être saisis et sur les démarches susceptibles de conduire à leur formation. On débouche ainsi sur les critères de validité de la sociologie et sur son insertion dans le mode de connaissances contenu dans tout système d'action historique. Dans une seconde partie, ces considérations théoriques sont appliquées à la société québécoise et à sa sociologie actuelle.
Tous droits réservés (C Les Presses de l'Université de Montréal, 1978
Ce document est protégé par la loi sur le droit d'auteur. L'utilisation des services d'Érudit (y compris la reproduction) est assujettie à sa politique d'utilisation que vous pouvez consulter en ligne.

https://apropos.erudit.org/fr/usagers/politique-dutilisation/ 


\section{Sociologie \\ mouvements sociaux conduites de rupture :}

\section{Le cas québécois}

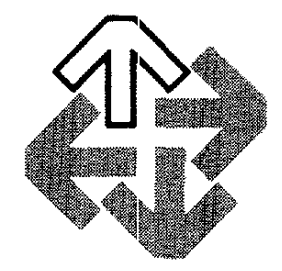

GABRIEL GAGNON

Si l'on postule, comme Alain Touraine, une liaison nécessaire entre sociologie et mouvements sociaux, il faut aussi s'interroger sur les méthodes par lesquelles ces mouvements sociaux peuvent être saisis et sur les demandes susceptibles de conduire à leur formation. À partir de ce problème, je tenterai d'amorcer une réflexion plus large sur les critères de validité de la sociologie et sur son insertion dans le mode de connaissance contenu dans tout système d'action historique.

Se voulant critique ou prolongement de l'approche de Touraine, ma réflexion la suivra donc d'abord d'assez près dans l'élaboration des concepts de «mouvement social» et de «mode de connaissance». A ce niveau, mes critiques devront beaucoup à un article d'Alberto Melucci ${ }^{1}$ qui servira de point de départ à plusieurs de mes interrogations sur les liaisons entre la sociologie et les systèmes d'action historique. Je m'aventurerai cependant, à l'aide d'auteurs comme Cornelius Castoriadis et Gilles-Gaston Granger, plus loin que les critiques prudentes de Melucci, avec tous les risques que comporte cette tentative.

1. Alberto Melucci, «Sur le travail théorique d'Alain Touraine", Revue française de sociologie, XVI, 1975, 359-379. 
Dans une seconde partie, je tenterai d'appliquer mes considérations théoriques à la société québécoise et à sa sociologie dans la conjoncture actuelle. Société dépendante mais libérale, soumise à des mouvements sociaux divergents, au seuil de la société programmée par son insertion nord-américaine, le Québec pose à la sociologie des problèmes spécifiques qui y donnent une coloration particulière à la position comme aux travaux des artisans de cette discipline. C'est alors que mes considérations théoriques devront révéler leurs limites comme leurs possibilités.

\section{LE SOCIOLOGUE COMME ACTEUR SOCIAL}

Je n'aborderai pas ici les distinctions faites par Touraine entre les sociologues qui travaillent au niveau organisationnel et institutionnel ou à celui du système d'action historique ou encore entre ceux qui choisissent la vulgarisation, l'éclat de la «lune académique» ou le statut d'intellectuel raté. Pour moi, la seule sociologie qui compte, la seule aussi qui pose vraiment les problèmes fondamentaux, c'est celle qui accepte de laisser la sécurité des dogmes et des idéologies et de se situer d'emblée au niveau le plus général, celui du système d'action historique, sans refuser cependant de considérer ses articulations possibles avec les autres niveaux.

Selon Touraine, le statut et les perspectives d'intervention d'une sociologie ainsi orientée sont bien loin d'être assurés. Elle doit se situer en effet à la jonction de deux perspectives apparemment contraires : un «attachement intransigeant aux exigences de la connaissance et le refus de la soumettre à l'idéologie» et, par ailleurs, «l'engagement auprès des collectivités sociales et nationales dominées et l'écoute des intellectuels qui dénoncent le rapport de domination ${ }^{2}$.»

Cette position difficile de la sociologie semble lui conférer à la fois un statut d'infériorité vis-à-vis d'autres disciplines plus indépendantes de l'acteur mais aussi une sorte de vocation méta-disciplinaire puisqu'elle ne peut résister à vouloir expliquer à la fois son domaine propre et sa façon de le connaître, devenant ainsi sa propre sociologie et sa propre épistémologie. Cette situation particulière, correspondant sans doute à l'état actuel d'une discipline encore en voie de libération des garants méta-sociaux, en particulier de celui fourni par l'économisme de la tradition marxiste, nécessite une réflexion attentive face aux critères de validation de la sociologie, que Melucci a bien amorcée. Je voudrais la poursuivre ici, non sans être conscient du caractère provisoire et hasardeux de mes considérations.

Melucci distingue quatre critères possibles de validation de la sociologie :

1. La validation formelle qui est «celle de la cohérence, de l'articulation systématique des concepts ».

2. Cette validation formelle est liée, comme l'a bien montré Kuhn, au consensus de la communauté scientifique qui en définit la configuration particulière à chaque période historique, constituant ainsi un second critère.

2. Alain Touraine, Production de la société, Paris, Seuil, 1973, p. 523. 
3. Le consensus de la communauté scientifique débouche sur la validation ernpirique, par voie de vérification ou de falsification. Cette forme de validation, concevable aux niveaux organisationnel et institutionnel, domaines de la sociologie traditionnelle, apparaît beaucoup plus difficile à appliquer lorsqu'on débouche sur le système d'action historique.

4. On peut alors être conduit à la validation par l'accord de l'acteur collectif, avec le risque de voir se transformer la science en idéologie ${ }^{3}$.

Four Melucci, lorsque Touraine affirme de la sociologie que «la validation de son analyse ne peut jamais être assurée par l'accord des acteurs intéressés», il la rejette inévitablement vers une auto-validation selon les deux premiers critères, vers une perspective wébérienne où la science apparaît comme une valeur en soi. Melucci, quant à lui, insisterait plutôt pour qu'on fasse dépendre les choix de recherche des fins qu'on attribue au sujet collectif avec qui on se sent solidaire et pour qu'on contribue à faire apparaître des pratiques qui réalisent les intérêts de cet acteur collectif qui serait la classe dominée ${ }^{3 a}$.

Cette discussion ne me semble pas faire avancer beaucoup le débat. Les deux protagonistes acceptent à la fois l'autonomie et la dépendance de la sociologie, Melucci rappelant cependant à Touraine que le sociologue, quoi qu'il dise, est partie du système d'action historique, qu'il est lui-même un acteur qui ne peut se situer, ne serait-ce que temporairement, au-dessus des rapports sociaux et des conflits.

Il me semble donc important d'introduire ici des remarques sur la troisième composante de l'historicité, avec l'accumulation et le modèle culturel, le «mode de connaissance», cette image de la société et de la nature propre à tout système d'action historique. Touraine parle assez peu de cette composante, qu'il considère «à la fois la plus fondamentale et celle qui jouera le rôle le plus limité dans l'analyse de la société».

Si elle est fondamentale, c'est «parce qu'elle manifeste le plus directement la capacité humaine de créer par la connaissance une image du monde, des relations sociales et du non-social».

Elle joue un rôle limité parce qu'elle n'apparaît que tout au début et à l'extrême fin de l'analyse. Elle semble se référer essentiellement au langage humain qui ordonne un univers déjà organisé par la technique, assurant ainsi le renversement de l'expérience sur elle-même qui permet à l'homme et à lui seul d'avoir une histoire», ou encore «au monde de l'image et du symbolique ${ }^{4}$ ».

Tout ce qui concerne le mode de connaissance est assez flou dans «Production de la sociêté». Empruntons encore à Melucci une définition qui essaie de préciser cette dimension du système de Touraine :

L.'action de la société sur soi suppose une capacitè symbolique, une faculté de connaissance qui organise le rapport à l'environnement par la constitution d' «états de nature», de définitions culturelles de la nature ${ }^{5}$.

3. Melucci, op. cit., p. 375-377.

3a. Ibid.

4. Touraine, op. cit., p. 28-32.

5. Melucci, op. cit., p. 362. 
On reconnaît ici la pensée de Moscovici où la nature devient une définition culturelle de la matière propre à chaque système d'action historique, ce qui amène à remplacer le terme «nature» par celui d' «états de nature» qui se réfèrent essentiellement à des systèmes de connaissance ${ }^{6}$.

Quel est le statut de la sociologie au sein de ce «mode de connaissance»? Il apparaît clairement chez Touraine que la société post-industrielle ou programmée est un nouveau système d'action historique par rapport auquel toutes les formations sociales, qu'elles y aient accédé ou non, ont à se définir et vers lequel elles semblent se diriger inéluctablement. Par ailleurs, alors que le XIXe siècle, encore trop soumis aux garants méta-sociaux, ne pouvait encore susciter de véritable analyse sociologique, la société programmée semble au contraire appeler ce type d'analyse, devenant le moment par excellence de la sociologie, grâce à l'apparition de nouveaux conflits impliquant des mouvements sociaux différents.

Cependant, pour le moment, ce qui caractérise le nouveau mode de connaissance de la société programmée c'est surtout «le grand développement des sciences naturelles de l'homme, de la biologie à la linguistigue et à l'anthropologie en passant par la psychologie génétique ${ }^{7} \gg$. Une bonne partie de la sociologie, surtout au niveau de l'analyse institutionnelle et organisationnelle, a été influencée par ce mouvement de naturalisation, dérivant ainsi du côté du pouvoir et des technologies sociales. Sur les rationalisations imposées à la nature et aux hommes par le développement de la société capitaliste, qui sont, au sens freudien, autant de camouflages de la réalité sous le couvert des idéologies, la sociologie vient poser son intellectualisation, nouveau langage scientifique et rationnel découpant autrement la réalité de façon à mieux mettre en évidence les conflits réels masqués par les rationalisations ${ }^{8}$. Cette tentative d'intellectualisation n'ayant pas encore complètement atteint, faute de méthodes et de théorie adéquates, le niveau du système d'action historique, elle y laisse libre cours aux constructions idéologiques trop liées aux points de vue des acteurs. C'est à ce niveau que, pour Touraine, le sociologue trouve son rôle principal : l'analyse des mouvements sociaux qui constituent pour lui un objet privilégié d'étude et d'intervention.

Ces mouvements sociaux, le sociologue en vivra, comme le sismologue des tremblements de terre. Pour qu'il puisse travailler en effet, il faut que l'action pratique de ces mouvements «attaque sans cesse la reproduction de l'ordre établi, défonce l'ordre social, fasse apparaître les rapports sociaux là où étaient installés les principes, les valeurs, les techniques ${ }^{9} \gg$.

Les mouvements sociaux sont «une action collective de classe, qui vise, par la mobilisation d'un acteur social contre un adversaire, à la réappropriation de l'historicité ${ }^{10} »$. Il est essentiel de retenir que, même s'ils se manifestent aux niveaux organisationnel et institutionnel, leur particularité est d'être «une visée

6. Serge Moscovivi, la Société contre nature, Paris, 10/18, 1972.

7. Touraine, op. cit., p. 521.

8. J'emprunte cette distinction qui me semble utile au chapitre VI de l'ouvrage de Fernand Dumont, les Idéologies, Paris, PUF, 1974

9. Touraine, op. cit., p. 514

10. Melucci, op. cit., p. 372. 
du système d'action historique à travers le conflit des classes ». La référence au conflit plutôt qu'à la contradiction implique d'une part que tout mouvement social se définit par rapport à un contre-mouvement avec lequel il partage un langage et un enjeu et d'autre part que son action est au niveau de la synchronie, contrairement aux autres formes de mouvements historiques que sont les actions critiques ou de rupture ou de mouvements de développement, tous deux situés dans la diachronie et visant un changement du système d'action historique.

Lorsque le sociologue veut saisir les mouvements sociaux ou les demandes susceptibles de conduire à leur formation, il doit rechercher deux types de phénomènes pouvant conduire à leur identification, au-delà des problèmes sociaux dont ils naissent. Ils se manifestent en effet par « une action collective durable, qui ne peut pas être réduite à des réactions à une crise ou à des tensions sociales particulières et aussi par une production idéologique ou utopique qui propose, même de manière fragmentaire, une vision de la société ${ }^{11}$ ". Le grand problème c'est que ces caractéristiques n'apparaissent souvent qu'à posteriori et ne permettent pas toujours de bien distinguer d'avance parmi les conduites collectives celles qui produiront le mouvement social.

En tentant de structurer ensemble pratiques concrètes et pratiques symboliques, le sociologue cherchera à composer, à dessiner un mouvement social, à en définir les acteurs et le véritable enjeu. N'apparaissant jamais à l'état pur, toujours masqués, leur organisation n'est jamais complète et leur conscience ne se développe pleinement qu'au moment où leur réalité s'estompe dans l'institutionnalisation.

Le sociologue vit des mouvements sociaux parce que son activité n'est possible que dans les sociétés qui laissent libre cours à l'émergence et au développement de ces mouvements. En un autre sens, c'est cependant lui qui leur confère leur réalité propre en les faisant apparaître comme objet sociologique au confluent des projets des acteurs et du système des rapports de classes. Le sociologue remplace en effet le langage des acteurs par le sien, langage construit par rapport au langage spontané, langage scientifique par rapport à l'idéologie et à l'utopie.

La validité de l'analyse deviendrait ainsi difficilement vérifiable empiriquement dans des formations sociales particulières, si les conflits fondamentaux et l'identité des acteurs des sociétés industrielles et programmées ne faisaient l'objet du consensus de leurs principaux observateurs.

De toute façon, si l'analyse du sociologue, le constituant lui-même en acteur, malgré sa prétention à la neutralité, n'amène pas à une nouvelle définition des conflits et de l'enjeu qui soit acceptée par d'autres acteurs sociaux, il sera passé à côté du mouvement social, il l'aura postulé là où il n'existait pas.

$\mathrm{Si}_{1,}$ au contraire, acceptée ou intégrée par les acteurs, l'intervention théorique du sociologue fait évoluer les conflits, en démasquant les idéologies et en identifiant les véritables rapports sociaux en jeu, l'opération conduit naturellement à l'institutionnalisation du conflit par le mûrissement, puis la disparition du

11. Touraine, op. cit., p. 407. 
mouvement social, dont la réalité floue a été intellectualisée, puis détruite par le scalpel du langage sociologique. Le sociologue devient ainsi, comme le linguiste ou l'anthropologue, celui qui au fond naturalise le social, institutionnalise l'effervescent. La sociologie vit sans doute des mouvements sociaux mais pour mieux les sacrifier ensuite en les dévoilant.

C'est que la sociologie est essentiellement liée, quoi qu'on en dise, au mode de connaissance de la société programmée. Comme les autres sciences de l'homme, son souci de validité scientifique l'oblige à intellectualiser les projets des acteurs comme les systèmes qui les encadrent, à appauvrir les significations des rapports sociaux en les soumettant au filtre de son langage spécifique. Qu'il le veuille ou non, qu'il en soit ou non conscient, que sa position l'associe ou non directement au projet technocratique, le sociologue, par son entreprise intellectuelle même, ne devient-il pas un des agents de la rationalisation du social portée par la bureaucratie post-industrielle ?

On comprend mieux ainsi les critiques adressées aux analyses des mouvements sociaux effectuées par Touraine, qu'il veut résolument synchroniques, l'analyse diachronique nécessitant d'autres concepts et une perspective différente. D'un côté, on n'est pas loin d'un certain structuralisme puisque le changement est évacué d'une analyse qui refuse délibérément de l'intégrer à ce stade. Par ailleurs, on voit comment, comme l'a bien montré Melucci, la validation par la science l'emporte finalement sur la validation par les acteurs. Le mode de connaissance, force productive dont fait partie la sociologie, n'est en effet pas considéré par Touraine comme relatif à un système d'action historique et par conséquent susceptible de modifications ou de dépassement sous l'effet des contradictions ${ }^{12}$.

Mais Touraine s'intéresse aussi au changement, même si ce qu'il en dit dans le chapitre VII de Production de la société demeure nettement moins convaincant que les analyses qui précèdent. Il se réfère essentiellement au concept de développement; employé de façon ambiguë comme «processus de passage d'une société d'un champ d'historicité à un autre». Il s'agit essentiellement chez Touraine du passage à la société programmée, considérée un peu comme un terme ultime, même si l'auteur admet que ce système d'action historique puisse aussi éventuellement être transformé.

Malgré les affirmations contraires, c'est un peu le même type d'analyse que pour la synchronie qu'on retrouve dans l'analyse du changement, les innovations, les mouvements de développement et les actions critiques venant s'ajouter aux mouvements sociaux et se définir par rapport à eux dans une logique qui se voudrait celle des contradictions mais qui se distingue assez mal de celle du conflit. Ainsi, un mouvement de développement est «un mouvement social orienté par un projet positif de changement sociétal, de développement et de libération"; quant à l'action critique ou de rupture, «il s'agit d'une révolte liée à une crise générale de la société ${ }^{13}$. «On ne peut ni séparer ni confondre

12. L'article de Louis Maheu, dans ce même numéro, abordera le problème de façon plus approfondie.

13. Touraine, op. cit., p. $449-450$. 
mouvement social et action critique. Ils sont les deux faces d'une protestation qui met en cause la domination sociale, les rapports du système d'action historique et des classes sociales ${ }^{14}$. » Selon que la rupture sera plus ou moins totale, on parlera d'action critique révolutionnaire, institutionnelle ou anticipatrice. Avec cette dernière, on retrouve l'intellectuel ou le sociologue, non plus cette fois comme détecteur de mouvements sociaux et de crises qu'il doit aider à venir à terme, en se tenant au-dessus pour les nommer, mais peut-être comme agent possible de changement du système. Se plaçant d'emblée au niveau des contradictions, il prend le risque de tomber dans l'utopie pour contribuer à une transformation du mode de connaissance qui aura des répercussions nécessaires sur le modèle culturel et l'accumulation. Dans cette tâche, le sociologue ne peut plus refuser de devenir acteur, le mode de connaissance devenant pour lui l'enjeu d'un conflit ${ }^{15}$.

Certains aspects de l'cuvre de Castoriadis ${ }^{16}$ me semblent permettre une critique encore plus fondamentale de la sociologie. Ce qui motive surtout cet auteur c'est le projet de dépasser les tentatives «scientifiques» d'intellectualiser ce qu'il appelle le «social-historique», qu'elles viennent du marxisme, du fonctionnalisme ou du structuralisme; toutes trois tributaires d'une logiqueontologie empruntée aux Grecs sans en faire une critique fondamentale, elles lui semblent parfaitement inadéquates pour expliquer véritablement l'histoire et le changement. Castoriadis suggère de recourir à ce qu'il nomme l'imaginaire social, fondement de toutes les institutions sociales comme des rationalisations scientifiques construites pour les expliquer :

De même chaque société définit et élabore une image du monde naturel, de l'univers où elle vit, en essayant chaque fois d'en faire un ensemble signifiant, dans lequel doivent trouver leur place certainement les objets et êtres naturels qui importent à la vie de la collectivité, mais aussi cette collectivité elle-même et finalement un certain «ordre du monde». Cette image, cette vision plus ou moins structurée de l'ensemble de l'expérience humaine disponible, utilise chaque fois les nervures rationnelles du donné, mais les dispose selon et les subordonne à des significations qui comme telles ne relèvent pas du rationnel (ni, du reste, d'un irrationnel positif), mais de l'imaginaire ${ }^{17}$.

Nous ne sommes pas loin du «mode de connaissance» tourainien sauf qu'il apparaît mieux ici dans sa relativité, sa précarité, comme un îlot temporaire au seir de l'imaginaire dans lequel il baigne et qui pourrait susciter sa transformation. Les précisions fournies sur la nature de cet imaginaire ne font que le rendre plus inaccessible aux méthodes habituelles de la sociologie, qu'il s'agisse de rationalisation idéologique ou d'intellectualisation scientifique :

Cet élément, qui donne à la fonctionnalité de chaque système institutionnel son orientation spécifique, qui surdétermine le choix et les connexions des réseaux symboliques, création de chaque époque historique, sa façon singulière de vivre, de voir et de faire sa propre existence, son monde et ses rapports à lui, ce structurant originaire, ce signifié-signifiant central,

14. Touraine, op. cit., p. 468.

15. Dans son dernier ouvrage, rédigé sous forme de journal, la Société invisible, Paris, Seuil, 1977, Touraine insiste de nouveau en plusieurs endroits sur la séparation nécessaire entre l'analyse des systèmes et des mouvements sociaux (synchronie) et celle du changement lié essentiellement à l'action de l'Etat (diachronie). La lecture du texte ne m'a cependant pas permis de répondre aux interrogations posées par Production de la société et exposées dans le présent article.

15. Je me suis surtout servi de Cornelius Castoriadis, l'Institution imaginaire de la société, Paris, Sleuil, 1975.

17. Castoriadis, op. cit., p. 208. 
source de ce qui se donne chaque fois comme sens indiscutable et indiscuté, support des articulations et des distinctions de ce qui importe et de ce qui n'importe pas, origine du surcroît d'être des objets d'investissement pratique, affectif et intellectuel, individuels ou collectifs cet élément n'est rien d'autre que l'imaginaire de la société ou de l'époque considérée ${ }^{18}$.

C'est sans doute à cet imaginaire latent que puisent les conduites de rupture, en manifestant l'existence sans jamais la dévoiler complètement. Le sociologue peut et doit être partie à cette opération si, comme nous l'avons souligné plus haut, il veut comprendre contradictions et changements. Malheureusement, ni Castoriadis ni d'autres n'offrent de méthode dite rigoureuse d'y arriver avec les instruments scientifiques produits jusqu'à maintenant par la sociologie.

Peut-être cet imaginaire, auquel la société programmée, débarassée de la rareté, serait susceptible de laisser plus de place, appelle-t-il un autre système d'action historique ${ }^{19}$, un autre mode de connaissance où la sociologie intellectualisante serait déplacée vers un retour à l'utopie, au sujet, à l'herméneutique $^{20}$.

Dans un article publié récemment, l'épistémologue Gilles-Gaston Granger, avec sa rigueur et sa subtilité habituelles, indique ce qui à mon avis pourrait être une façon de mieux fonder les intuitions de Castoriadis ${ }^{21}$. Cherchant ce qu'il nomme la rigueur philosophique, pour en montrer la possibilité et la nécessité par rapport à la rigueur scientifique, Granger décrit les concepts philosophiques comme des méta-concepts manifestant les significations au-delà des objets, pour fonctionner «essentiellement comme métalangue à l'égard des énoncés exprimant des connaissances objectives, et de ceux qui se rapportent aux diverses facettes de la pratique ${ }^{22}$ »: l'analyse effectuée à l'aide de ce langage "part du symbolisme comme d'un fait; à la différence de la science qui détruit et réforme ce symbolisme naturel en vue de constituer un monde d'objets exactement associable à des énoncés bien formés, la philosophie s'attache à expliciter le rapport des symbolismes - y compris de ceux que construit la science - à l'expérience qu'ils organisent de façon latente ou manifeste, organisation virtuelle qui du reste n'est actualisée justement que par l'exposition philosophique, dont on doit dire en ce sens qu'elle n'a pas d'objets, ne crée pas d'objets, mais qu'elle élabore des significations ${ }^{23}{ }$. J'ai l'impression de retrouver ici, sous le nom d'analyse philosophique, cet aspect essentiel de la tâche du sociologue, qui, acceptant de mettre en question les certitudes de son langage habituel, fourni par le mode de connaissance propre à la société programmée, plonge du côté de la rupture, de l'utopie, de l'imaginaire à la recherche non plus de nouveaux conflits dont il pourrait être le juge et l'ordonnateur au dessus ou à côté des acteurs mais d'un nouveau mode de connaissance et d'un nouveau système d'action historique où il sera inéluctablement dans la mêlée avec tous les risques pour l'acteur impliqués par l'imprévisibilité de la diachronie ${ }^{24}$.

18. Castoriadis, op. cit., p. 203.

19. Pourrait-il s'agir de la société auto-gestionnaire?

20. Il faut songer ici à l'œuvre du philosophe Ernest Bloch et à ses utopies concrètes.

21. Gilles-Gaston Granger, «Les concepts philosophiques», dans la Philosophie et les savoirs, Montréal, Bellarmin, 1975, p. 21-74.

22. Granger, op. cit., p. 69.

23. Granger, op. cit., p. 68.

24. Les réflexions de Granger sont reprises de facon plus sociologique dans «Sur le traitement comme objets des faits humains», dans Savoir, faire, espérer : les limites de la raison, Bruxelles, Publications des Facultés universitaires Saint-Louis, 1976. 
Cette tâche demeure beaucoup plus difficile que celle de saisir les mouvements sociaux en synchronie où l'on dispose toujours de l'analyse des systèmes pour mettre en perspective les acteurs et intellectualiser leurs rationalisations, et où le sociologue peut aussi se permettre de tenir pour acquis et non soumis aux rapports sociaux le mode de connaissance dont il tire sa légitimité et ses instruments d'intellectualisation. Et pourtant, si dans nos sociétés avancées, le mode de connaissance est du côté des forces productives, le sociologue ne peut éviter d'y devenir un acteur autour des enjeux que constitue l'appropriation sociale de sa discipline comme la forme d'intellectualisation qu'elle impose aux relations sociales comme aux langages qui les rationalisent. C'est peut-être en ce sens que le sociologue ne peut s'en tenir à une référence wébérienne aux facteurs internes de validité de sa discipline mais se trouve toujours projeté du côté des acteurs dont qu'il le veuille ou non, il fait inéluctablement partie. Ni Touraine ni Melucci ne me semblent donc être allés assez loin dans leur critique de la sociologie, craignant au dernier moment d'abandonner un ultime garant méta-social.

Si j'ai tenté d'élargir de façon exploratoire le débat théorique sur les critères d'une sociologie se voulant fondamentalement critique, c'est pour mieux éclairer le cas de la sociologie québécoise auquel je voudrais maintenant m'intéresser de façon particulière.

\section{DU CÔTÉ DE LA SOCIOLOGIE QUÉBÉCOISE}

Mes hypothèses seront ici nécessairement fragmentaires et intuitives, puisqu'elles se veulent non pas réflexion sur l'histoire de la sociologie québécoise, très bien élucidée par Fournier ${ }^{25}$, mais reflet d'une conjoncture où mouvements sociaux et perspectives sociologiques s'entremêlent d'autant plus que le Québec partage encore le statut de société dépendante.

Je trouve ici particulièrement utiles les considérations de Touraine sur les sociétés dépendantes, non pas tellement celles de Production de la société mais celles qu'il applique dans Vie et mort $d u$ Chili populaire où la richesse de l'analyse concrète donne son véritable sens à une théorie pas toujours assez rigoureusement construite selon moi ${ }^{26}$.

L'idée que, dans les sociétés dépendantes, des mouvements sociaux démembrés et multiples constituent une réalité intermédiaire entre mouvements sociaux simples et actions critiques, m'apparaît fondamentale. Il en est de même pour les trois axes des rapports sociaux distingués dans le cas du Chili ${ }^{27}$ : l'axe classe populaire - classe dirigeante, l'axe nation dépendante - nation dominante et l'axe organisation traditionnelle - organisation moderne, dont la conjonction est nécessaire pour comprendre toute formation sociale dépendante. $\mathrm{J}$ 'expliquerai plus loin de quelle façon on doit modifier ce modèle de base pour tenir compte du statut de dépendance politique du Québec et de sa participation à la société programmée nord-américiane. Mon analyse, différente de celle de

25. Marcel Foumier, Institutionnalisation et différenciation de disciplines dans une situation de double dépendance, E.P.H.E., thèse de doctorat de $3^{\mathrm{e}}$ cycle, Paris, 1973.

26. Alain Touraine, Vie et mort du Chili populaire, Paris, Seuil, 1973.

27. Touraine, ibid., p. 116. 
Paul Bélanger et Céline Saint-Pierre présentée ailleurs dans ce numéro, pourra, je l'espère, apporter une perspective complémentaire, propice à l'approfondissement de la discussion.

Je n'entrerai pas dans les polémiques qui cherchent à savoir si le Québec constitue ou non une formation sociale susceptible d'une analyse spécifique. À mon avis, le statut de dépendance politique du Québec, se conjuguant à sa situation au centre de l'économie mondiale ${ }^{28}$ et aux différences linguistiques et culturelles qui distinguent sa population, implique qu'on doive le considérer comme une formation sociale tronquée n'ayant pas encore atteint son plein épanouissement. Ceci justifie une analyse qui tienne aussi bien compte de sa situation et de ses rapports de classes spécifiques que de son accès à la société programmée via l'insertion au capitalisme nord-américain.

Dans un article récent de «Sociologie et sociétés», Jules Savaria considère à juste titre le Québec comme une région retardée du «centre» :

La formation québécoise apparaît bien déterminée par un développement auto-centré, assumé non par le capital local, mais par le capital américain et anglo-canadien auquel le capital autochtone est associé à titre mineur. Le Québec est donc région du centre et région en voie de développement (vs en voie de sous-développement), mais région mineure ou retardée parce que dominée ${ }^{29}$.

En effet, le Canada et le Québec n'ont jamais fait partie de la périphérie, au sens qu'Amin donne à ce terme. Ils ont constitué ensemble un «centre jeune» qui a réalisé sa première industrialisation à partir de petits producteurs indépendants, le processus s'effectuant à des époques différentes selon les régions, pour amener graduellement l'ensemble de la population à la consommation de masse : il s'est ainsi constitué une bourgeoisie nationale essentiellement ontarienne, c'est-à-dire anglophone. À la suite de la seconde guerre mondiale, une partie de cette bourgeoisie, s'appuyant sur l'Etat fédéral canadien, semble avoir opté pour cette industrialisation restrictive et internationalisée que Cardoso a bien décrite dans le cas du Brésil ${ }^{30}$. Certaines régions, comme les quatre provinces maritimes, le Manitoba et la Saskatchewan et aussi le Québec, semblent avoir été laissées pour compte par ce processus d'intégration poussée à l'économie américaine, demeurant liées à l'ancienne structure industrielle adaptée à un stade antérieur du capitalisme.

Les conséquences de ce type d'industrialisation se répercutèrent sur les rapports de classe et l'ensemble de la société, dans le sens prévu par Cardoso :

Une scission de la structure sociale, de type vertical, s'ajoute à la scission horizontale. Ainsi non seulement les entrepreneurs se diviseront selon qu'il s'agit d'un groupe s'orientant vers le modèle de développement national ou vers le modèle d'industrialisation restrictive et internationalisée, mais aussi les autres groupes sociaux s'articulent en fonction de cette coupure. Il y aura un prolétariat moderne, lié aux entreprises à productivité élevée qui offrent à relativement peu d'employés des niveaux de rémunération corrects, et un prolétariat traditionnel, une petite bourgeoisie technico-professionnelle et une classe moyenne traditionnelle; même des institutions comme l'État, les Forces Armées ou l'Université, auront

28. Je me réfère ici à la terminologie utilisée par Samir Amin. Voir en particulier Samir Amin, le Développement inégal, Paris, Minuit, 1973.

29. Jules Savaria, «Le Québec est-il une société périphérique?», Sociologie et sociétés, vol $7, \mathrm{n}^{\circ} 2$, novembre 1975.

30. Fernando Enrique Cardoso, Politique et développement dans les sociétés dépendantes, Paris, Anthropos, 1971. 
des centres d'orientation du comportement polarisés différemment selon les deux modèles en question ${ }^{31}$.»

La «Révolution tranquille» au Québec, processus lent dont la manifestation politique principale est la venue au pouvoir du Parti libéral en 1960, peut s'interpréter dans cette perspective, comme une tentative d'adaptation à un nouveau mode de développement. En modernisant les institutions politiques et l'organisation sociale, le groupe au pouvoir pouvait espérer mieux s'intégrer au nouveau stade du capitalisme et peut-être créer un pendant québécois à la nouvelle bourgeoisie canadienne en formation.

Féussie, si on peut employer ce terme, au niveau de la modernisation des systèmes de santé et d'éducation, cette entreprise ne put parvenir à constituer autour de l'État québécois une bourgeoisie susceptible de profiter directement, sans l'intermédiaire de la bourgeoisie canadienne, de l'intégration à l'économie nord-américaine. On assista alors à un clivage à l'intérieur de cette bourgeoisie étatique en formation, un groupe choisissant d'aménager le statut de double domination ${ }^{32}$, l'autre optant pour une indépendance politique à long terme qui aurait au moins pour effet d'éliminer un des intermédiaires ${ }^{33}$.

C'est ainsi que s'est constitué, à l'intérieur même du mouvement d'intégration au capitalisme américain, l'axe conflictuel nation dominante-nation dépendante au sein de la formation sociale canadienne. Le Parti québécois, par sa composition et son programme, semble susceptible de faire passer au statut de bourgeoisie étatique une "petite bourgeoisie» de type nouveau formée grâce à la démocratisation poussée du système d'éducation et au développement des secteurs public et para-public de l'économie. Dans un ouvrage fort bien documenté ${ }^{34}$, Maurice Saint-Germain montre en effet comment, quant à la répartition de la valeur de la production et de l'emploi entre secteurs primaire, secondaire et tertiaire, l'économie québécoise partage avec celles du Canada et des États-UUnis plusieurs des caractéristiques inhérentes à l'ère post-industrielle.

Le projet de la petite bourgeoisie québécoise nécessite cependant un renforcement substantiel de l'Etat provincial qui le met en concurrence directe avec une bourgeoisie financière et industrielle canadienne soucieuse de se servir de son propre État pour renforcer son intégration aux multinationales au sein de l'espace économique nord-américain, tout en gardant sous sa domination l'espace économique québécois. Depuis sa venue au pouvoir, une conjoncture économique défavorable et le souci d'obtenir une majorité importante au référendum ont empêché le Parti québécois d'adopter des politiques significatives allant dans cette direction.

C'est parallèlement à cette opposition fondamentale entre fractions de la bourgeoisie pour le contrôle de l'accumulation que s'inscrit le deuxième axe

31. Cardoso, op. cit., p. $159-160$.

32. Il s'agit du Parti libéral reporté au pouvoir avec Robert Bourassa en 1970, après une éclipse de quatre ans, puis battu par le Parti québécois aux élections du 15 novembre 1976.

33. Le Parti québécois, né en 1968 d'une scission au sein des artisans de la «Révolution tranquille», a depuis sa venue au pouvoir en 1976, opté pour la thèse édulcorée de la «souverainetéassocialion * qu'il espère faire endosser par l'électorat à l'occasion d'un référendum qui devrait être tenu en 1979.

34. Maurice Saint-Germain, Une économie à libérer. Le Québec analysé dans ses structures économiques, Montréal, Les Presses de l'Université de Montréal, 1973. 
de conflit, dissocié du premier, même s'il participe du même processus d'accumulation et du même modèle culturel. Il s'agit du conflit classe des travailleursclasse dirigeante. Jusqu'à l'avènement de la «Révolution tranquille», ce conflit se livrait surtout entre des entreprises contrôlées par des capitaux américains ou canadiens anglophones et un syndicalisme dit international, c'est-à-dire composé de filiales des syndicats américains. Depuis 1960, avec le développement des secteurs public et parapublic et leur syndicalisation, l'axe principal du conflit s'est déplacé vers une opposition entre l'État québécois et les syndicats des travailleurs ouvrant dans les domaines de l'éducation et de la santé, regroupés surtout dans les centrales exclusivement québécoises que sont la Confédération des syndicats nationaux (CSN) et la Centrale des enseignants du Québec (CEQ). Cette évolution du conflit de classes entraîne sa radicalisation, les revendications syndicales rejoignant, à travers les priorités budgétaires de l'État, la société globale; le conflit fait aussi éclater la dimension nationale, le syndicalisme voyant dans le Parti québécois au pouvoir l'État patron alors que ce dernier craint de céder le contrôle de son projet de société à la classe des travailleurs. Une bonne partie de la classe ouvrière regroupée dans la Fédération des travailleurs du Québec (F.T.Q.), continue cependant à se déterminer face aux entreprises multinationales et au capitalisme restrictif dont parle Cardoso, reproduisant un clivage assez semblable à celui qui divise la bourgeoisie ${ }^{35}$. Nous demeurons quand même toujours, qu'il s'agisse de l'opposition nation dominante - nation dépendante ou de celle entre travailleurs et classe dirigeante, à l'intérieur du même système de modernisation, du même modèle culturel, le partage des fruits de l'accumulation étant le principal enjeu du conflit entre les divers acteurs.

Il nous reste à aborder le troisième axe de rapports sociaux suggéré par Touraine pour l'analyse des sociétés dépendantes, celui de la modernisation où l'organisation moderne est confrontée à la société traditionnelle. C'est ici que le cas du Québec prend une coloration particulière, due au fait que la société programmée $^{36}$ y occupe déjà une place importante. Deux formes principales d'opposition à une modernisation représentée autant par les Partis libéral et québécois que par le mouvement ouvrier se sont manifestées au Québec ces dernières années. Le mouvement créditiste, forme de populisme issue des secteurs mis en danger par la modernisation (agriculteurs, ouvriers des industries traditionnelles, producteurs indépendants dans le domaine des services), au delà d'une certaine insistance sur la défense de la famille et de la religion, exprime le mieux politiquement une réaction du «petit» face au «gros», qu'ils soient représentés par les syndicats bureaucratisés, les écoles régionales polyvalentes, le ministère des affaires sociales ou les sociétés multinationales ${ }^{37}$. La seconde forme de résistance à la modernisation vient d'une «contre-culture » qui a pris au

35. La situation se complexifie davantage si l'on sait que les travailleurs du secteur public ont tendance à voter massivement pour le Parti québécois tout en combattant syndicalement l'État qu'il anime alors que ceux du secteur privé appuient les politiques économiques de l'État tout en divisant leurs suffrages entre les partis libéral et québécois. 1969.

36. Sur cette notion, voir Alain Touraine, la Société post-industrielle, Paris, Médiations,

37. On pourra consulter sur ce mouvement Gabriel Gagnon, «Populisme et progrès » Recherches sociographiques, vol. XVIII, $n^{\circ} 1$, janvier-avril 1976, p. 23-34. G. Gagnon, A. Sicotte et $G$. Bourassa, Tant que le monde n'ouvrira pas les yeux ou les Créditistes de la Beauce vus par euxmêmes, Montréal, Quinze, 1977. 
Québec, chez les jeunes des milieux urbains, une expansion particulière à la suite d'une scolarisation accrue accompagnée de la croissance du chômage des diplômés : essentiellement apolitique, ce mouvement insiste sur la libération individuelle, au delà du marxisme et du capitalisme. Ces deux mouvements de résistance, opposés autant qu'il se peut dans l'idéologie de leurs acteurs, partagent cependant objectivement un certain nombre de thèmes qu'on pourrait rapprocher du «small is beautiful» de Schumacher ${ }^{38}$.

Certains phénomènes apparus récemment semblent indiquer d'ailleurs une alliance possible entre ces deux perspectives, les jeunes scolarisés adeptes de la nouvelle culture se retrouvant au travail au sein des secteurs défavorisés urbains ou ruraux, avec les chômeurs et les assistés sociaux, à la recherche d'un nouveau modèle culturel et peut-être d'un nouveau mode de connaissance qui pourrait esquisser le passage à ce nouveau système d'action historique que constituerait sans doute la société autogestionnaire. En milieu urbain, ceci s'est concrétisé partiellement dans la création en 1974 d'un nouveau parti municipal, le Rassemblement des citoyens de Montréal (RCM) qui, malgré les efforts de certains de ses membres pour l'entraîner vers une idéologie soit purement nationaliste soit purement marxiste-léniniste, essaie de donner voix à un mouvement de lutte urbaine où les nouveaux thèmes de l'écologie et de la restructuration des unités sociales de base constituées par les quartiers pourraient être dominants. En milieu rural, dans une région comme le Bas-du-Fleuve et la Gaspésie dont les 300000 habitants sont soumis depuis 1963 aux séquelles de l'important travail de planification effectué par le Bureau d'aménagement de l'Est du Québec (B.A.E.Q.), commence à s'exprimer une convergence encore plus significative entre ruraux traditionnels (pêcheurs, agriculteurs, bûcherons) menacés par la rationalisation du secteur primaire et nouveaux venus issus de la contre-culture des villes et ayant choisi le retour à la campagne. C'est là que des mouvements qu'on appelle «Opérations Dignité » ont, par leur action politique, arrêté à douze cas la fermeture prévue d'environ quatre-vingts villages; on y retrouve côte à côte anciens et nouveaux ruraux, le «savoir-faire» technique des premiers s'unissant à la technologie sociale des seconds pour contrer la rationalité purement économique au nom du maintien d'un «mode de vie» ou de la promotion de la «qualité de vie». Fait significatif, certains des nouveaux ruraux ont remplacé les leaders traditionnels, qui étaient surtout des curés, à la direction des organisations économiques et politiques spécifiques issues de ce mouvement global dont l'influence idéologique dépasse les limites de la région ${ }^{39}$. Ces deux cas montrent la complexité des conflits autour de la modernisation dans le cas du Québec, puisqu'ils suggèrent à la fois retour au passé et création d'une société nouvelle de type post-industriel. Ces mêmes ambiguités affectent d'ailleurs toutes les tentatives d'élaboration d'un socialisme autogestionnaire.

J'ai finalement été entraîné à poursuivre beaucoup plus loin que je ne l'aurais souhaité ici l'analyse des mouvements sociaux québécois. Ce déblayage m'était cependant nécessaire pour étayer ce qu'il me reste à dire sur la sociolo-

33. E.F. Schumacher, Small is Beautiful, London, Abacus, 1974.

39. Cf. Charles Banville, les Opérations dignité, Québec, Le Fond de recherches forestières de l'université Laval, 1977. 
gie québécoise, sur les positions sociales de ceux qui la pratiquent et sur les principales caractéristiques de leurs travaux et de leurs interventions.

Les sciences sociales au Québec, particulièrement la sociologie à laquelle ses méthodes permettent moins pour le moment de s'abstraire des projets des acteurs, loin de constituer un univers à part défini exclusivement par des critères de validité interne, sont éminemment liées à l'univers des mouvements sociaux du fait du statut de dépendance occupé par le Québec tant dans l'économie nord-américaine que dans le champ scientifique international. L'imbrication de la sociologie dans les conflits spécifiques de notre situation de dépendance apparaît ici beaucoup plus claire que dans les sociétés dominantes où son statut scientifique et son institutionalisation peuvent permettre un certain camouflage de ses liaisons aux rapports de classes.

Un court article de Céline Saint-Pierre sur cette question nous paraît la simplifier un peu trop, du moins en ce qui concerne la sociologie, en distinguant «trois axes principaux qui sous-tendent les analyses produites par les chercheurs en sciences humaines :

- «celles qui reposent sur le statu quo, redoublent l'idéologie dominante et contribuent à camoufler la réalité sur laquelle est fondée la société québécoise, c'est-à-dire le capitalisme monopoliste et l'impérialisme américain.

- «celles qui rendent compte directement des intérêts de la classe à laquelle appartient la majorité des intellectuels québécois; elles servent directement leurs intérêts de petits-bourgeois individualistes, réformistes et nationalistes.

- «celles qui sont le produit d'intellectuels progressistes cherchant à s'aligner sur les positions du prolétariat avec comme objectifs premiers de dévoiler dans toute leur complexité les structures fondamentales de la société et de démasquer les antagonismes propres au capitalisme ... fondés sur les contradictions qui ne peuvent être réglées que par une transformation radicale, révolutionnaire du mode de production capitaliste ${ }^{40}$ ».

Il va de soi qu'au Québec, Fournier l'a montré dans sa thèse ${ }^{41}$, la principale tâche de la sociologie a été de se libérer de la philosophie thomiste qui l'avait fait naître et prétendait continuer à fournir les normes devant régir ses analyses concrètes. Pour ce faire, elle dut s'appuyer à la fois sur la tentative de rattrapage institutionnel et politique culminant dans la « Révolution tranquille» et sur les techniques de mesures soi-disant neutres fournies alors par une sociologie américaine très orientée vers les problèmes de modernisation. Cette première libération est bien exprimée de l'intérieur dans l'itinéraire sociologique de Guy Rocher ${ }^{42}$ qui fut un des premiers à l'effectuer de cette façon pour influencer ensuite de nombreux chercheurs. La sociologie se mit alors tour à tour, sous le couvert de la neutralité, au service des projets de modernisation et de rationalisation prônés d'abord par la «Révolution tranquille» à Québec puis par l'équipe du manifeste "pour une politique fonctionnelle ${ }^{43}$ " venue au pouvoir à Ottawa avec Trudeau à partir de 1965.

40. Céline Saint-Pierre, «Les sciences humaines servantes du pouvoir ou de la population ", Chroniques, vol. $1, \mathrm{n}^{\circ} 6-7$, juillet 1975 , p. 117-121.

41. Fournier, op. cit..

42. Dans Recherches sociographiques, vol. $\mathrm{XV}, \mathrm{n}^{\circ} 2-3,1974, \mathrm{p} .243-248$.

43. A. Breton, R. Breton, C. Bruneau, Y. Gauthier, M. Lalonde, M. Pinard, P.E. Trudeau, «Pour une politique fonctionnelle», Cité libre, mai 1964, p. 11-17. 
C'est dans la mouvance de ce type de sociologie que de nombreux postes de fonctionnaires ont été occupés à Québec et à Ottawa alors que se consolidait le développement institutionnel des départements de sociologie de Québec et de Montréal, de nombreux étudiants venant y préparer la carrière promise par l'avènement des nouvelles technologies sociales.

Le B.A.E.Q., dont j'ai parlé plus haut, constitue un excellent microcosme du tra.vail des sociologues de cette période. Cet organisme joua un rôle central dans l'orientation de notre discipline : d'abord il constitua au Québec une des premières formes de recherche appliquée à grande échelle, impliquant, à un titre ou à un autre, une bonne partie des sociologues québécois de cette époque et, par ailleurs, parce qu'en essayant, par l'animation sociale, de rejoindre les populations, il tentait à sa manière de détecter les mouvements sociaux potentiels et de contribuer à leur émergence.

Le recours trop exclusif, faute d'alternative, aux théories américaines de la modernisation, convaincues d'une voie unique et nécessaire vers le développement, comme le souci de produire rapidement un plan cohérent, amenèrent finalement le B.A.E.Q. à ne faire de l'animation qu'une sorte d'agitprop destinée à vendre les idées des planificateurs; ainsi ses travaux aboutirent plutôt: à la rationalisation du secteur primaire de l'économie et à l'exode d'une partie de la population qu'à la création d'une société régionale viable et autosuffisante.

Le type de liaison sociologie-société amorcé par le B.A.E.Q. entre 1963 et 1966 se poursuivit ensuite sur deux axes opposés à partir des conflits dégagés par cette expérience.

La tentative de rationalisation du social se déplaça de la région du Bas-duFleuve vers le gouvernement du Québec où plusieurs des principaux protagonistes du B.A.E.Q. vinrent essayer de mettre en vigueur leurs politiques au niveau de la société globale. Cet assaimage important coïncida avec le processus de nationalisation du champ scientifique dans le secteur des sciences sociales, bien décrit par Maheu et Fournier ${ }^{44}$, alors que l'État québécois intensifie son intervention en particulier dans les secteurs de la santé, du bien-être et de l'éducation et que son effort de financement de la recherche dans ces domaines devient à peu près équivalent à celui des organismes fédéraux. Cette évolution s'effectue à partir d'une sociologie de type fonctionnaliste, venant s'adjoindre aux techniques de rationalisation des choix budgétaires (PPBS) aussi implantées.

Une telle sociologie n'est donc plus neutre, ni directement liée au champ scientifique canadien ou américain, contrairement à ce qui se passe encore dans les sciences fondamentales, mais plutôt aux intérêts des diverses couches de la bourgeoisie, indépendantiste ou non, cherchant à s'assurer une assise politique autour de l'État québécois.

Plusieurs des sociologues initiés aux techniques de l'animation sociale durant l'expérience du B.A.E.Q. y avaient, quant à eux, découvert le conflit et la lutte des classes au-delà de la participation et de la modernisation, devenant

44. Louis Maheu et Marcel Fournier, «Nationalismes et nationalisation du champ scientifique québécois $»$, Sociologie et sociétés, vol. VII, n² 2, novembre 1975. 
ainsi conscients de l'échec fondamental de cette entreprise dans une perspective de transformation globale de la société. Le milieu rural leur apparaissant peu propice au développement des conflits fondamentaux, ils déplacèrent en milieu urbain, surtout montréalais, leurs techniques et leurs préoccupations. À partir d'organisations de quartiers, de comités de citoyens et de partis politiques embryonnaires, rejoignant surtout les assistés sociaux et les travailleurs nonorganisés, ils tentèrent de faciliter l'émergence du conflit travailleurs-classe dirigeante. Cette naissance d'un mouvement social urbain amena graduellement à la réappropriation d'un marxisme-léninisme jusque là assez absent des débats, parallèlement à l'établissement à l'Université du Québec à Montréal (UQAM) de plusieurs départements de sciences sociales carrément orientés, celui de sociologie surtout, dans une perspective théorique marxiste. Le processus d'imitation, souvent détecté dans les sociétés dépendantes, n'est cependant pas absent des efforts des sociologues marxistes québécois. Empruntant directement leurs concepts et leurs analyses au renouveau du marxisme français, ils donnent souvent l'impression de les plaquer sur la réalité québécoise ou encore, en optant pour l'optique de la reproduction, de refuser toute médiation vers le changement qui ne serait pas transformation globale à partir d'un parti des travailleurs encore inexistant. Cette perspective globale risque bien souvent de tomber dans un stérile académisme de gauche en évitant de s'insérer dans la complexité des mouvements existants. En voulant s'aligner sur un prolétariat encore sans voix politique, le sociologue s'expose à sombrer dans une idéologie désincarnée, aussi éloignée de la science que des mouvements sociaux réels.

Le gros de la sociologie québécoise m'apparaît donc coincée dans la double dépendance scientifique envers la France et les États-Unis bien analysée par Fournier ${ }^{45}$; cette situation fournit à la fois un alibi scientifique au processus de rationalisation du projet étatique québécois, dans le cas de la sociologie américaine et au mouvement pour la création d'un parti des travailleurs, dans le cas de la sociologie française. A mon avis, aucune de ces deux perspectives, si on lui accorde l'exclusivité qu'elle réclame, ne peut cependant rendre compte de l'ensemble de la formation sociale québécoise, des mouvements sociaux qui s'y affrontent et des contradictions susceptibles d'amorcer sa transformation.

Le Québec n'est pas en effet qu'un Etat dominé en voie d'émancipation ou qu'une société industrielle en proie au conflit bourgeoisie - prolétariat. Il est tout cela mais aussi fortement soumis à l'emprise de la société programmée en formation, avec les nouveaux problèmes, conflits et contradictions que ce processus entraîne. Quelques sociologues québécois ont essayé de se mettre dans cette perspective pour construire une sociologie critique qui accèderait aux conduites de rupture, pour détecter les processus de transformation pouvant mettre en cause le statut même de la sociologie marxiste ou fonctionnaliste. Cette sociologie embryonnaire essaie de dépasser de diverses façons le langage et les techniques habituelles de la discipline, soit du côté du vécu, soit du côté des structures cognitives, soit du côté de la poursuite de l'imaginaire social, dans le sens des positions esquissées à la fin de la première section de cet article.

45. Fournier, op. cit. 
Ein ce qui concerne le vécu, mentionnons le programme proposé récemment par la revue Recherches sociographiques :

L'objectif des sciences humaines n'est pas de superposer au vécu des constructions qui le remplaceraient, mais d'en rendre compte dans des interprétations qui ne le dépassent qu'en l'assumant. Il ne s'agit pas pour autant de dissoudre l'explication dans les «mystères» de l'expérience des agents sociaux, mais d'aller assez creux dans cette expérience pour être assuré que la remontée théorique reste fidèle à ce dont elle doit rendre compte ... Aussi faut-il rappeler que notre objet est historique, qu'il change comme la sociologie que nous en faisons, et d'habitude plus vite que cette dernière. Revenir au vécu, ce n'est donc pas chercher quelque terreau de la conscience intemporelle mais bien plutôt se souvenir que la sociologie reprend toujours les tentatives des agents sociaux eux-mêmes pour se donner une vision d'ensemble de la situation où se déterminent leurs comportements ${ }^{46}$.

Une recherche à base d'histoires de vie, portant sur les mutations du Québec depuis 1940, s'inscrit dans cette perspective. Analysées en fonction des catégories sociales utilisées par les informateurs, de leur conscience historique, de leur mobilité et, de façon plus globale, de leur type de «vécu» ou d'insertion symbolique et pratique dans la société, ces histoires de vie permettent de dépasser les ornières de l'analyse de contenu quantitative pour essayer d'atteindre les transformations objectives déjà connues d'une société, à partir des représentations des acteurs à un moment donné du temps. Le but visé ici est essentiellement cependant la reconstitution du contenu culturel de la conscience de claisse.

Une méthode d'approche différente, celle de Patrick Donovan, consiste à appliquer les techniques de l'ethnoscience et de la "cognitive anthropology" dans un village de la Beauce, en y tenant compte de la stratification sociale, contrairement à ce que font ordinairement les anthropologues. Il s'agit encore ici, à partir du mode de connaissance des acteurs, de dépasser les intellectualisations du sociologue pour le rendre plus conscient non seulement des contenus exprimés par les discours des habitants de Saint-Côme mais aussi des structures inconscientes, mentales ou affectives, qui les fondent ${ }^{47}$.

Plus ambitieuse, une autre recherche, celle de Marcel Simard, veut, du point de vue du vécu et de l'imaginaire social, analyser l'expérience des travailleurs de Tricofil, à Saint-Jérôme au Québec, qui sont passés en quelques années du syndicalisme d'affaires au syndicalisme de combat puis à une autogestion menacée. Ce chercheur essaie de dégager les significations et le potentiel de transformation sociale de ce qu'on peut appeler une conduite de rupture potentielle face au système économique dominant. C'est ici autant du côté de l'imaginaire castoriadicien que de celui du vécu ou des structures mentales que s'effectue le dépassement des sociologies traditionnelles. Les projets des acteurs demeurent encore insérés cependant dans l'analyse du système des rapports de classes ${ }^{48}$.

C'est finalement Marcel Rioux qui, parmi les sociologues québécois, pouse le plus loin la théorisation de ces nouvelles perspectives, ancrées dans les

46. Fernand Dumont et Nicole Gagnon, «Le vécu : présentation», Recherches sociographiques, vol. XIV, ${ }^{\circ} 2,1973$, p. 153-154.

4.7. Cette recherche fait l'objet d'une thèse de doctorat au Département de sociologie de l'Université de Montréal : elle devrait être déposée au cours de l'année 1978.

48. Cette recherche sera elle aussi présentée dans une thèse de doctorat au Département de sociologie de l'Université de Montréal au cours de l'année 1978. 
mouvements d'opposition au modèle culturel et même au mode de connaissance dominant que nous avons décrits plus haut. Esquissée dans un itinéraire sociologique $^{49}$, cette réflexion s'est poursuivie au cours d'une recherche sur l'aliénation et la vie quotidienne dans les quartiers de Montréal et devrait déboucher bientôt sur la publication d'un important essai théorique ${ }^{50}$. Pour Rioux, la professionnalisation de la sociologie participe au «parachèvement par le haut du grand enfermement dont le mode de production capitaliste a besoin pour se déployer»; la recherche des pratiques nécessaires de destructuration de la société comme de la sociologie devra donc se faire autant du côté du «monde ordinaire», des chansonniers et de la vie quotidienne que de celui des piquets de grève ou du travail des scientifiques ${ }^{5 t}$.

Le problème, lorsqu'on s'aventure du côté des «sentiers de la création», consiste cependant à pouvoir distinguer ce qui signifie rupture et contradiction et ce qui n'est que récupération ou reproduction camouflée, ce qui augure transformation réelle du mode de connaissance et ce qui ne fait que le renforcer. Comme nous l'avons esquissé plus haut, sortir du synchronique et des mouvements sociaux, où l'on dispose de critères plus sûrs d'interprétation, c'est risquer les impasses cognitives et pratiques de la poursuite d'utopies riches sans doute de pouvoir de transformation du système d'action historique mais aussi de possibilités de fuite définitive hors du champ des conflits sociaux réels. La tentative demeure quand même nécessaire si l'on veut atteindre la complexité de la réalité québécoise et y inventer une sociologie qui ne soit pas qu'une copie conforme de ce qui a été fait ailleurs : notre statut hybride de société dépendante entrée dans l'ère post-industrielle nous contraint d'ailleurs à cette tâche spécifique.

Je ne prétends au terme de cette analyse ni avoir fourni une critique définitive des concepts de «mode de connaissance» et de «mouvement social» ni avoir inventé une nouvelle façon de voir la sociologie québécoise. Tout au plus, en soulignant certaines failles des travaux déjà entrepris sur ces sujets, ai-je cru pouvoir contribuer aux débats en cours.

Sans avoir critiqué, comme l'a fait Louis Maheu, la dichotomie société programmée - société industrielle, que j'ai acceptée d'emblée sans chercher à en approfondir la véritable nature, j'en suis quand même arrivé comme lui à mettre en doute la possibilité d'expliquer fondamentalement le changement à l'intérieur du système d'Alain Touraine. À mon avis, cette carence provient surtout de son refus de soumettre aux rapports de classes cet élément essentiel du système d'action historique qu'est le mode de connaissance ${ }^{52}$. Partant de là, on comprend mieux aussi pourquoi Touraine a pu arriver à situer le sociologue d'une certaine façon au-dessus des acteurs, les critères de validité de son entreprise demeurant plutôt ceux de l'univers scientifique, comme l'a montré Melucci ${ }^{53}$.

49. Dans Recherches sociographiques, vol. XV, $\mathrm{n}^{\circ}$ 2-3, 1974, p. 311-312.

50. Marcel Rioux, Essai de sociologie critique, Montréal, HMH (sous presse); Yves Lamarche, Marcel Rioux et Robert Sévigny, Aliénation et idéologie dans la vie quotidienne des Montréalais francophones, Montréal, 2 vol., Les Presses de l'Université de Montréal, 1973.

51. Rioux dans Recherches sociographiques, ibid.

52. Je me réfère à l'article de Louis Maheu publié dans ce numéro.

53. Melucci, op. cit. 
ILa seule issue à ce recours à d'ultimes garants méta-sociaux m'est apparue résider dans la soumission de ce «mode de connaissance» à l'analyse en termes de conflits et d'enjeu, débouchant sur une critique fondamentale de sa forme et de son contenu au delà du langage de la sociologie traditionnelle et au risque de le mettre lui-même en question.

Par ailleurs, l'analyse de la situation spécifique de la société québécoise, de ses mouvements sociaux et de sa sociologie, m'apparaît ouvrir certaines possibilités d'aller dans ce sens en dépassant marxisme et fonctionnalisme, à la poursuite des conduites de rupture et des utopies produites par notre situation de société dépendante hautement développée. Cette voie demeure encore cependant semée d'embûches.

Je rejoindrai encore ici les analyses de $\mathrm{Maheu}^{52}$ en prétendant que notre type de société, qu'il soit spécifiquement différent ou simple avatar du capitalisme, est gros d'un nouveau système d'action historique en gestation, société autogestionnaire dont l'avènement s'ancrerait autour du «mode de connaissance» devenu principale force productive. Les sociologues n'y pourraient échapper au statut d'acteurs privilégiés de la lutte des classes.

\section{RÉSUME}

Postulant avec Touraine une liaison nécessaire entre sociologie et mouvements sociaux, l'auteur s'interroge sur les méthodes par lesquelles ces mouvements peuvent être saisis et sur les démarches susceptibles de conduire à leur formation. On débouche ainsi sur les critères de validité de la sociologie et sur son insertion dans le mode de connaissances contenu dans tout système d'action historique. Dans une seconde partie, ces considérations théoriques sont appliquées à la société québécoise et à sa sociologie actuelle.

\section{SUMMARY}

Postulating, as does Touraine, that a necessary connection exists between sociology and social movements, the author reflects on methods by which these movements can be understood and on the steps likely to lead to their formation. This leads us to criteria of validity for sociology and to its insertion into the form of knowledge contained in any system of historical action. In a second part, these theoretical considerations are applied to Quebec society and to sociology in Quebec today.

\section{RESUMEN}

Postulando con Touraine, una ligazón necesaria entre Sociología, y movimientos sociales, el autor se interroga sobre los métodos por los cuales esos movimentos pueden ser comprendidos y sobre las gestiones suceptibles de conducir a su formación. De este modo desemboca sobre los criterios de validez de la Sociología, y sobre su incersión en el modo de conocimientos contenidos en todo el sistema de acción històrica. En una seguanda parte esas consideraciones teóricas son aplicadas a la sociedad quebequense y a su sociología actual. 